\title{
Effective FE models for simulating the elasto-mechanical characteristics of forming machines
}

\author{
Robert Tehel $^{1} \cdot$ Thomas Päßler $^{1} \cdot$ Markus Bergmann $^{1}$
}

Received: 11 June 2019 / Accepted: 12 December 2019 / Published online: 6 January 2020

(C) The Author(s) 2020

\begin{abstract}
The elasto-mechanical characteristic of forming machines is crucial for a variety of forming processes. Numerical simulations are to be performed to understand the complex interaction between process, forming tool, and machine. The machine's elastic fingerprint depends on its design, accuracy of the components, and mounting conditions. Models only based on data of the machine design are limited. Therefore, effective FE models are to be developed to transfer the physical structure into a digital representation. The paper presents different methods of creating effective models for the characteristics of forming machines determined by measurements. Examples and potential of using these models are presented.
\end{abstract}

Keywords Finite element method (FEM) $\cdot$ Machine behavior $\cdot$ Forming machines

\section{Introduction}

\subsection{Elasto-mechanical characteristics of forming machines}

In the field of metal forming, various aspects affect the progress and quality of a forming operation. In order to obtain a complete mapping of the process via simulations, a steadily increasing number of these aspects are modeled within the simulations. Especially common simulation environments for forging and stamping lead to realistic results for the behavior of the workpiece during the process with respect to the given die geometries [8].

In this context, special emphasis is placed on the boundary conditions of forming simulation, or the forming dies. Common forming simulations refer to a rigid behavior of the underlying tool geometries [1], particularly when the focus remains on the interaction among the tool's active components. Many forming simulation environments offer the usage of elastic deformations for the dies, since regarding

Robert Tehel

robert.tehel@iwu.fraunhofer.de

1 Fraunhofer IWU, Reichenhainer Straße 88, 09126 Chemnitz, Germany the tools, compliancy yields an increase in accuracy as exemplarily probed by Neto et al. [7] or Pilthammar et al. [11] for drawing processes and by Chen et al. [4] for a stamping process. Furthermore, this step demands only little effort, since the exact geometry of the forming tool is available as CAD data. In most cases, the fixed supply for the clamping surface of upper and lower die remains a global boundary condition for the simulation.

Some studies of Großmann et al., Pilthammar et al., or Schongen et al. $[6,10,12]$ have shown positive effects of additionally integrating the elastic behavior as well as the stiffness behavior of the interacting machine components, such as press table and press ram (including ram guidance as well as the drive line), as outlined in Fig. 1a. The importance of these aspects is also stated by Groche et al. [5] with their approach of measuring and manipulating the stiffness and the compliancy of the interacting press components.

Contrary to the existing geometrical data for the forming tool, generally no usable data for the behavior of an existing forming machine exists in a form directly usable for the tool manufacturer. Even with CAD data of the machine, either from the manufacturer or achieved by reverse engineering, it stands to reason whether the behavior of the model would approximate the real deformations sufficiently. Especially for older forming presses, as most of the machines in the field are, there is no CAD data accessible. Thus, one of the most essential problems consists of creating models of 


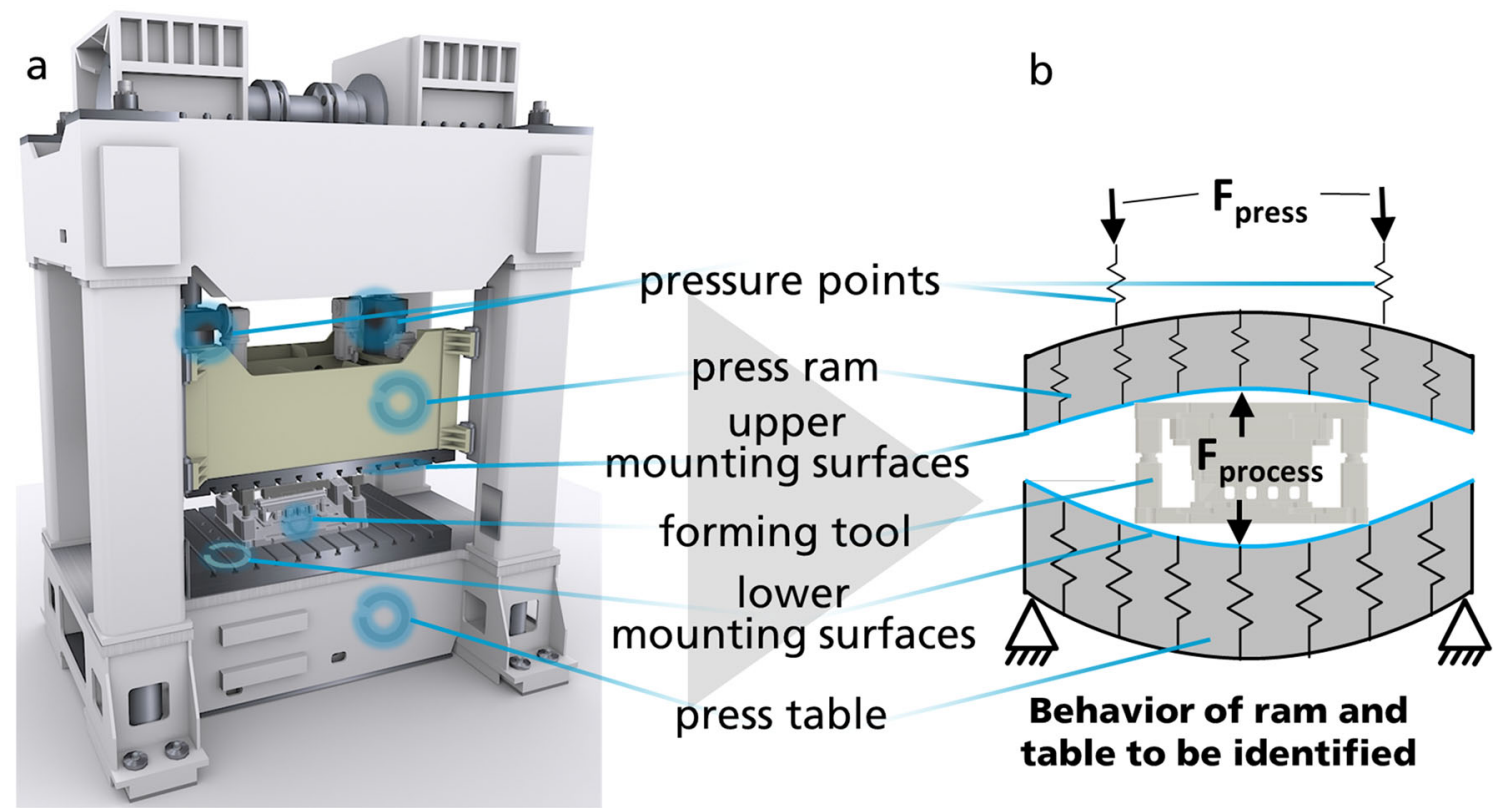

Fig. 1 Sheet metal forming press with die-press interaction zone (a) and schematic free body diagram of elastic components (b)

the elasto-mechanical behavior of the forming machine's relevant components out of practical measurements.

This more promising approach of generating FE models for reproducing the elastic deflection of the machine due to external loading out of measured data from the individual machine in its actual state is the target of this paper. The main options for such an approach are the parametrization of analytical models as mentioned by Penter [9] and the fitting of phenomenological models [10, 13].

For forging processes, an inline simulation may be sufficient, with analytical models representing the elasticity of the machine $[2,3,12]$. In the case of sheet metal, forming this approach is questionable due to the increased complexity of the process and the strong implications of small geometrical deviations.

\subsection{Objective and advancement}

This paper aims for a solution of a model based on measured data, generated as a phenomenological model with a base that is preconditioned by prior knowledge. The model claims to be of sufficient accuracy and, concurrently, to operate unsusceptibly against varying load scenarios while having only a small impact on the overall size of the simulation model. Due to the increasing complexity and number of parameters, the accuracy has to be limited to reach these other goals. This does not have to be a disadvantage when accuracy is leveled with the general uncertainties of the forming tool manufacturing process, the variation in the press behavior, the tool mounting conditions, or variance of the workpiece blank, including its material [15].

\section{Measuring the elastic behavior}

\subsection{Requirements for the measurement}

The measurement is intended to characterize the elastic behavior of ram and table of the press. Therefore, it is necessary to measure deflections corresponding to the respective load states. The elastic deformations have to be gathered in as many locations as possible, and preferably evenly spread over the clamping surfaces of ram and table. The load state has to be applied in a way that can be reproduced identically in the FE simulation. The press has to be in the actual configuration which the FE model shall represent, i.e., the configuration has to be defined regarding the pressure points, adapter plates, etc. Further, the load has to be applied at different load levels and for different load distributions, representing smaller and larger tools as well as operations with low and high forces.

\subsection{Methods for data acquisition}

The data for the presented models results from the measurement of displacements with a laser tracker, as shown in Fig. 2. It is gathered by determining the global coordinates of a number of target points at the press both for the unloaded and the loaded state of the machine and by determining their difference. The resulting differences are total deformations corresponding to a specific load distribution measured with load cells (usually four) and a summed total press force [13]. Other options for measuring elastic deformations include optical camera devices [10], 
Fig. 2 Investigated press with 4 load cells (a), laser tracker (b), and measuring points (c) on press table (d) as well as press $\operatorname{ram}(\mathbf{e})$

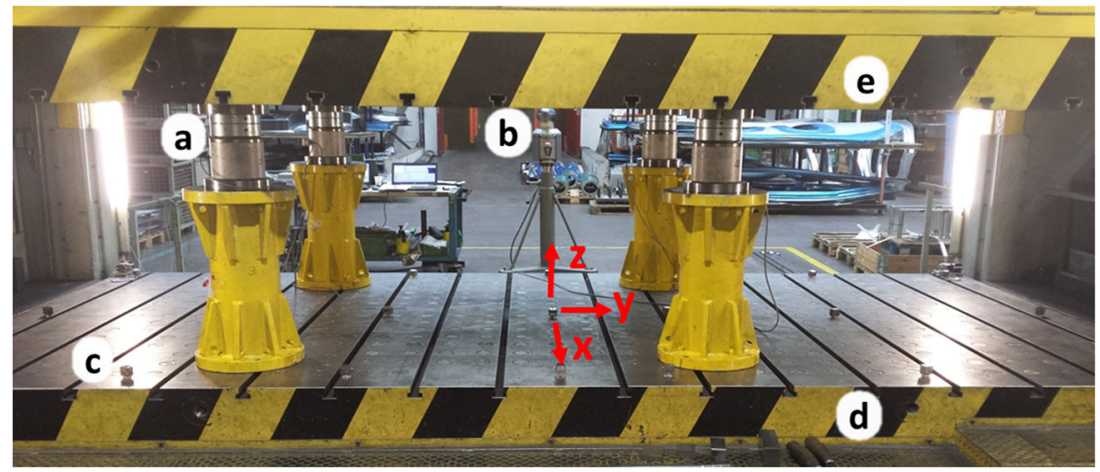

tactile sensors on a measurement framework [14], or dial gauges. The advantage of using the laser tracker in combination with single load cells on pillars lies in the high flexibility of the system, the accuracy of measurement, and the inertial coordinate system.

\subsection{Measured deformations and handling of non-linearities}

The flatness of clamping surfaces as measured, for example, in Fig. 3, is one origin of non-linearities and functions as an offset to the elastic deflection of the press, since occurring gaps between forming tool and press table as well as press ram will be closed when increasing the press force.

Most of the deviation in flatness results from plastic deformation of the forming machine during its lifetime. When considering multiple machines, these plastic deformations are statistically random, contrary to the elastic behavior of the forming machines which is determined by their structural design.

In Fig. 3, deviations are discretized in a 50-mm pattern to increase reliability and reduce local effects being too small to affect the forming process.

The same applies to uneven clamping surfaces of the forming tool. However, these values are normally negligible since the surfaces are newly machined.
When considering the elastic behavior of the press or the deflections of the clamping surfaces of press ram and press table, the functional interrelationship is not linear. Measurements were carried out regarding the vertical deflection as a function of total press force and position (Fig. 4). The elastic behavior can be linearized sufficiently for higher loads since the non-linearities are majorly located at lower press force. Therefore, it is necessary to measure under different load levels for each configuration.

\section{FE models of the elasto-mechanical characteristic}

\subsection{Requirements for the FE models}

The FE models have to represent the correct elastic behavior of the elastic press components independent from which forming tool is surveyed in the simulation, including its individual load distribution. Since this paper focuses on the press table and the press ram as the most relevant elastic components, two individual models have to be prepared in such a way, that they can be combined with a forming tool in the forming simulation. Since the deformation of an elastic component depends strongly on the induced load distribution, it is not sufficient to generate an effective FE
Fig. 3 Flatness of clamping surface of an unloaded press table

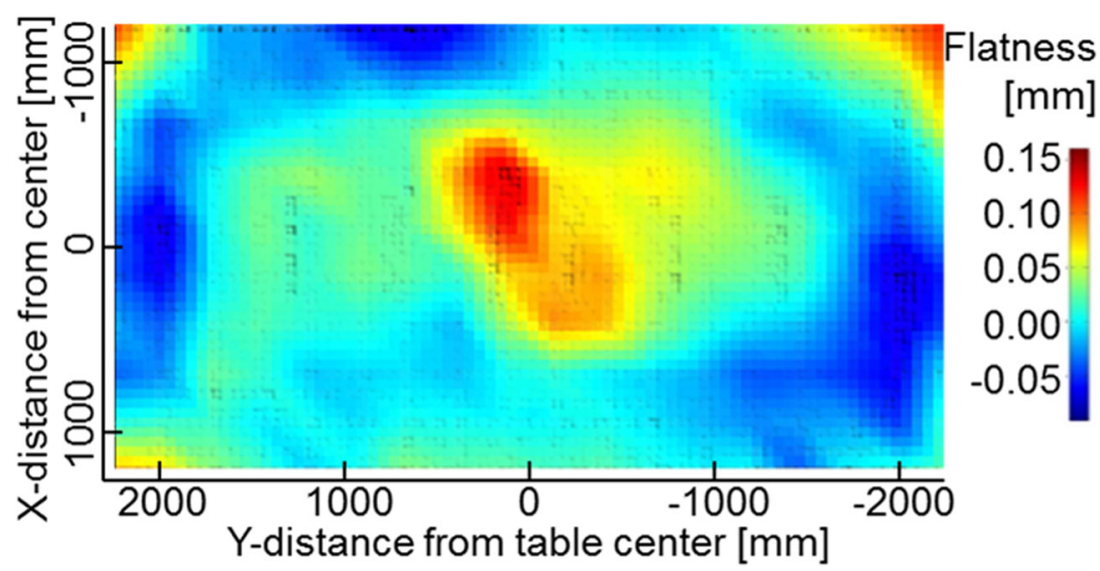



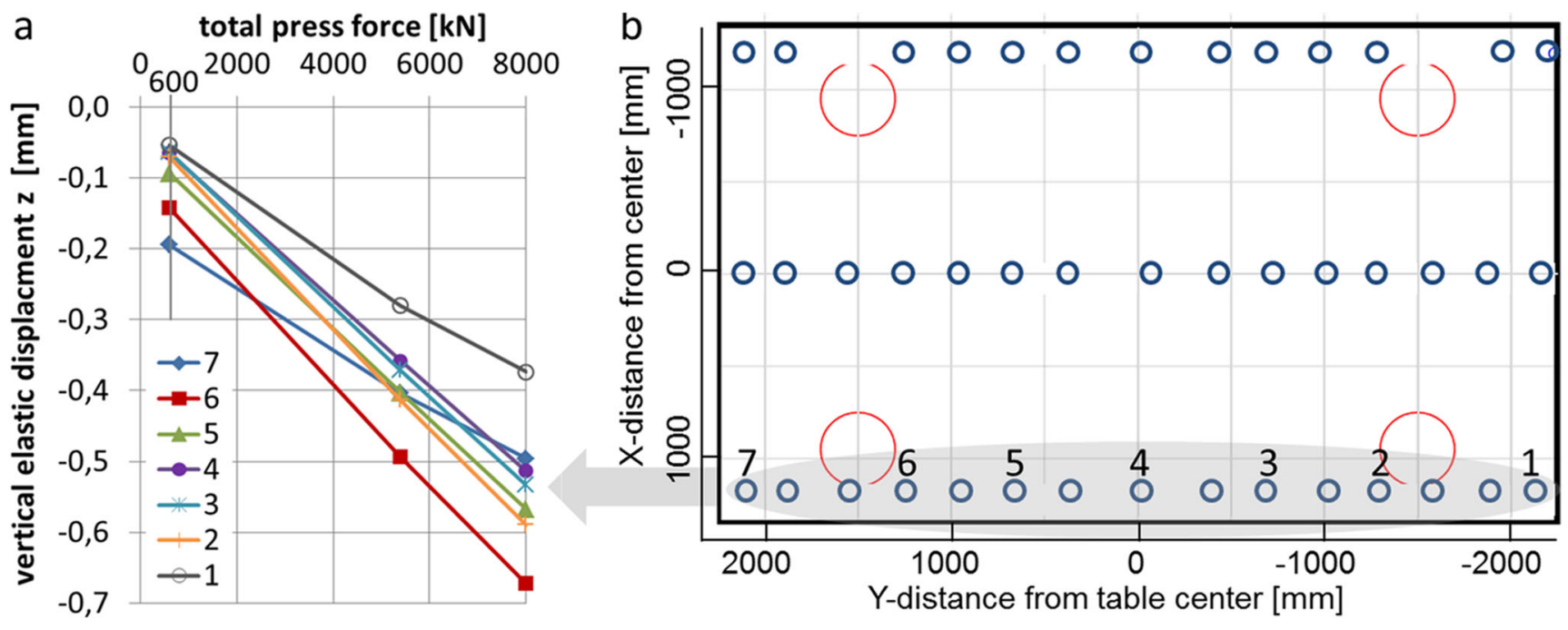

Fig. 4 Measured vertical elastic displacements as a function of total press force (a) and position on press table (b)

model from a single measured load case. Instead, the FE model has to be generated or optimized using a set of different load cases, which ideally covers the variety of theoretically possible distributions of loads induced during the forming process.

In practice, this requirement has to be reduced to the most typical load distributions. As stated before, forming tools cannot be used at this point since the pressure distribution cannot be digitized sufficiently. A reduction to single forces measured by load cells is a recommendable approach to reproduce the experimental load scenario via boundary conditions in the FE simulation. Approachability of the clamping surfaces is an additional advantage. The measurement and evaluation of these reduced load distributions are justified for an elastic behavior following the principle of superposition that is given for the approximation of the two considered elastic components.

\subsection{Modeling approach based on topological optimization}

Commonly, topological optimization is used to enhance material efficiency during a design process by eliminating areas with low loads. Thus, the stiffness-to-weight ratio can be optimized. The available framework for the adjustment of Young's modulus $E_{j}$ for each individual FE element $j$ is used in this approach for modeling the elastic behavior of a press table. Boundary conditions include the measured loads (via load cells) together with fixed support in the area of the press foundation. The optimization of $f\left(E_{j}\right)$ as shown in Eq. 1 is the minimization of the residuum between measured deformation $\hat{u}_{i}$ at measuring position $i$ and the corresponding simulated one $u_{i}$.

At this stage, the model would be optimized for a single measured load case, which deviates strongly from the claim to obtain a universal model working for various load scenarios $p$.

$f\left(E_{j}\right)=\min _{E} \sum_{i=1}^{n}\left(u_{i}-\hat{u}_{i}\right)^{2}$

$f\left(E_{j}\right)=\min _{E} \sum_{p=1}^{m} \sum_{i=1}^{n}\left(u_{i, p}-\hat{u}_{i, p}\right)^{2}$

It is essential to integrate all available load cases $p$ into one optimization function $f\left(E_{j}\right)$ with the same set of optimized elements elastic moduli of $E_{j}$, as seen in Eq. 2. After this stage, the model is in principle capable of emulating all required load cases. When joining multiple measured load cases in one optimization procedure, the need for weighting arises to define where the inevitable inaccuracy is tolerable, which is caused by the higher compression of information in the same model. It can be used to define the importance of a load case and the varying relevance of regions on the clamping surface and to compensate for the effects from different press forces for different load cases. Equation 3 contains the weighting factor $a_{i, p}$.

$f\left(E_{j}\right)=\min _{E} \sum_{p=1}^{m} \sum_{i=1}^{n} a_{i, p}\left(u_{i, p}-\hat{u}_{i, p}\right)^{2}$

Figure 5 a shows a cuboid as the most universal starting point for the iterative topological optimization of a press table under press forces $F_{1}$ to $F_{4}$ and with fixed support. Here, the mounting plate is a separate body in the press table assembly, so it can be excluded from the optimization to ensure valid contacts with the tool later on and to turn the deflection of the clamping surface into a continuous function $C_{1}(x, y)$. The elements close to the fixed support are also excluded from optimization. 

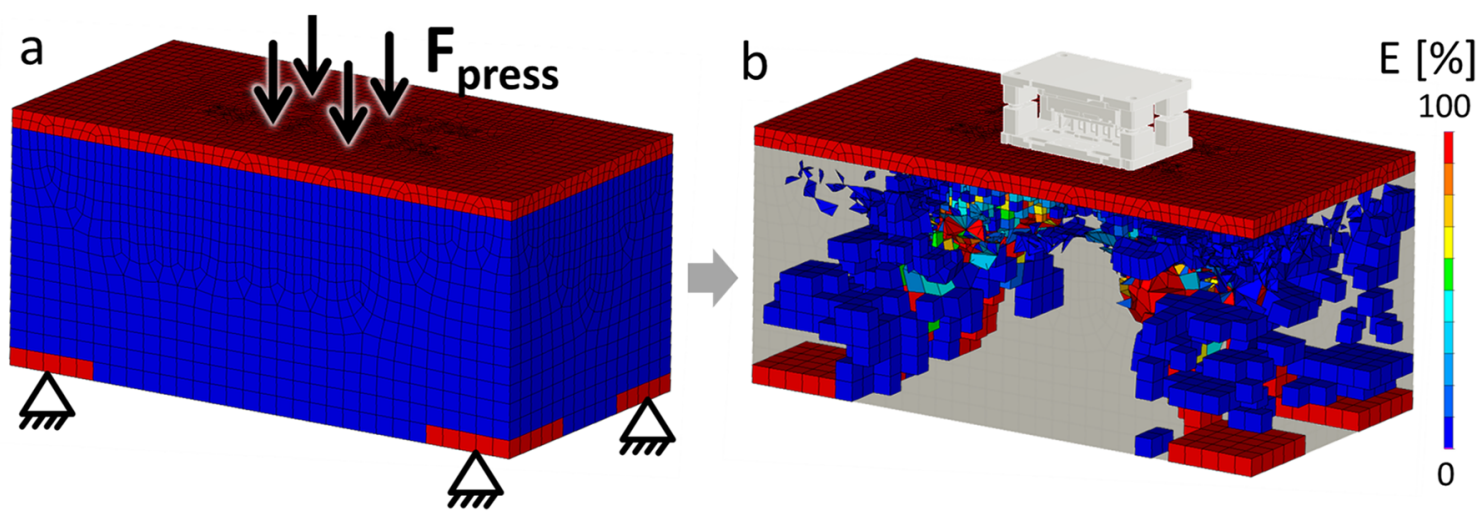

Fig. 5 Cuboid model with optimization domain (blue) and interaction zones (red) (a). Resulting distribution of optimized Young's modulus (b)

Figure $5 \mathrm{~b}$ shows the resulting model of the press table with optimized Young's moduli for each element. Here, Young's modulus decreases from 100\% (red) to 0\% (blue), and elements with a modulus close to zero are masked out. Due to the large dependence among neighboring elements, optimization of this model is not efficient. Numerous elements that are highly linear-dependent are required due to the large starting volume, so that optimization takes a long time even when using larger elements.

Using the above knowledge on the basic architecture of a common press table, the cuboid starting model can be defined in a form that is easier to optimize. Figure 6 illustrates this approach, combining the generic model of the press table with its mirror twin as the press ram together with a forming tool. The geometries for the two models of press table and press ram consist of optimizable sparse bodies (light part) and clamping plates with mounting surfaces that are not optimized (dark part). For the optimizable parts of the models, the intended mesh is already shown and illustrates the linear independent elements, which are rather large compared to the dimension of the structure.

The single elements are much more independent due to the thinned out structure with three layers parallel to the clamping surfaces (for distributing shear and bending), and a truss framework made of single element links between them. Thus, in addition to the strongly reduced number of elements, the convergence of the optimization is highly improved, which together results in reduced computation time. The accuracy of the model in reproducing the measured deflections for the measured load cases is still high, as shown for an exemplary hydraulic press in [13]. Another advantage of the model generated by this strategy is the decreased overhead in the simulations combined with the forming tool geometry and the forming operations.

Further, Fig. 6 shows the simple interconnection between the model of the forming tool and the established models for the press ram and press table. This is secured by the continuous clamping surfaces of the bolster plates, which are not optimized.

\subsection{Modeling approach based on parameter optimization}

Although the boundary condition for the press table is quite robust, the relatively flexible support of the ram leads to poor convergence and accuracy of the topological
Fig. 6 Combination of forming tool together with topologically optimized sparse models of press ram and press table

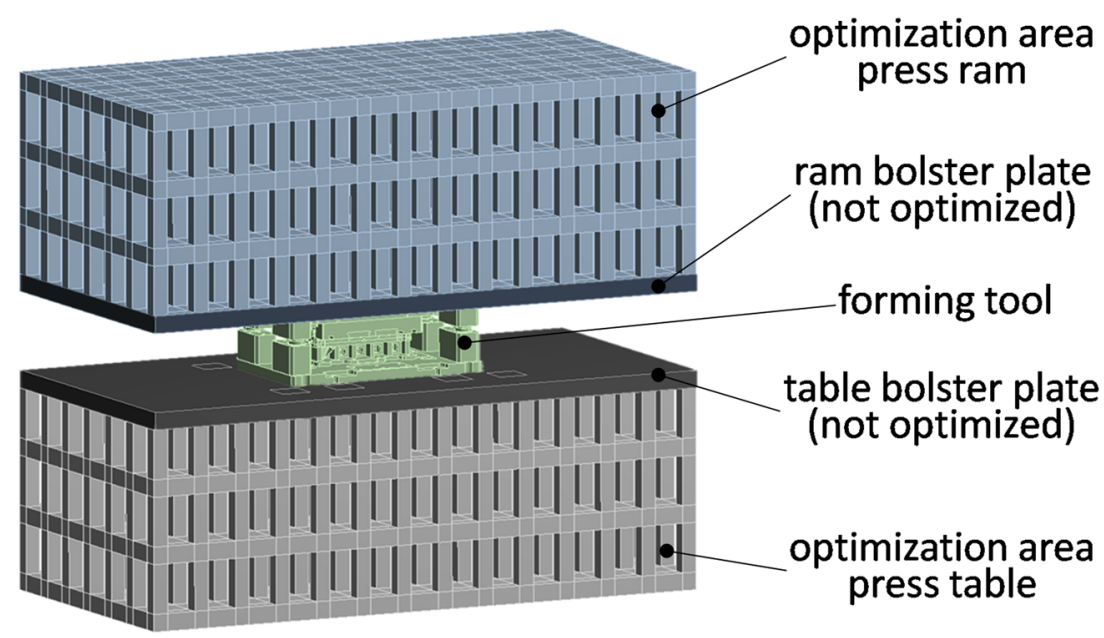


optimization of the press ram. This is also based on the simple formulation of the available objective function Eq. 3 in the used software Simulia Tosca®. Thus, Fig. 7a shows another approach for modeling the elastic behavior of the press ram.

To overcome the restrictive objective function in topological optimization regarding only displacements, a modeling strategy based on parameter optimization is used for the press ram. Especially when using the least squares method, it is possible that neighboring evaluation points deviate in opposite directions, which drastically increases the inclination error, as illustrated in Fig. 8. Since the inclination is of nearly the same importance as the deflection itself but cannot be covered with the topological approach, the new optimization strategy is developed.

A model for the press ram is generated from shells (clamping surface, longitudinal and transverse web plates) and discrete vertical springs, as shown in Fig. 7a. This model has a significantly lower number of free parameters. The low number of parameters representing shell thicknesses and discrete uniaxial stiffnesses allows in addition to the reduced simulation time to outsource the optimization in a Matlab® routine. In this routine, the objective function can be defined freely and in a current state involves three terms for the deflections and the inclinations.

$$
\begin{aligned}
f\left(k_{j}\right)= & \min _{k} \sum_{p=1}^{m} \sum_{i=1}^{n} a_{i, p}\left(u_{i, p}-\hat{u}_{i, p}\right)^{2} \\
& +b_{i, p}\left(\left.\frac{\partial u}{\partial x}\right|_{\left(x_{i, p}, y_{i, p}\right)}-\left.\frac{\partial \hat{u}}{\partial x}\right|_{\left(x_{i, p}, y_{i, p}\right)}\right)^{2} \\
& +c_{i, p}\left(\left.\frac{\partial u}{\partial y}\right|_{\left(x_{i, p}, y_{i, p}\right)}-\left.\frac{\partial \hat{u}}{\partial y}\right|_{\left(x_{i, p}, y_{i, p}\right)}\right)^{2}
\end{aligned}
$$

Equation 4 is an adaptation of Eq. 3 with the new optimization parameters $k$ involving the discrete springs and the shell thicknesses. Further, it includes additional terms for considering the inclination errors as illustrated in Fig. 8.
The error of displacement at evaluation point $i$ during load case $p$ is weighted with $a_{i, p}$; the inclination error in $x$-direction at the same point is weighted with $b_{i, p}$, and the inclination error in $y$-direction is weighted in accordance with $c_{i, p}$. The weighting, which is generally independent of any physical property, results from the importance of the individual evaluation points, where central points are more relevant for the interaction with the forming tool. Good convergence can be assured by using this general approach for ram modeling, where the fixed support of the springs includes the characteristic of the pressure points. Tilting can be adjusted via the boundary conditions.

When attaching the uniaxial springs directly at single nodes, the behavior of the model becomes partly dependent on the mesh size. Since the model is individual for each analyzed forming machine, the mesh size can be set according to the demand of the simulation as well as to the size of the machine. For the modeling of the measured test presses with clamping surfaces in the size of $2500 \mathrm{~mm} \times 4500 \mathrm{~mm}$, the mesh size for the model of the ram was defined to $50 \mathrm{~mm}$. The number of parameters to be optimized results out of the 45 uniaxial springs together with the 7 shell thicknesses.

\subsection{Linearization and integration of non-linearities in the model}

While the presented reduced models exhibit linear elastic behavior, the measured deflections are nonlinear depending on the total press force, as illustrated in Fig. 4. For small ratios of the nonlinear parts on the total displacements, they may be neglected; otherwise, they have to be considered in the model.

One option is to add the nonlinear parts to the linear ones, which takes no additional effort, but leads to a model that is accurate only for exactly one press force, i.e., the one from the measurement. For smaller press forces, the simulated deformations will be too small since the nonlinear offset is underestimated, and for larger press forces the
Fig. 7 Model of press ram made of shells and discrete vertical springs for parameter optimization (a). The same model with FE mesh together with model of press table out of topological optimization (b)
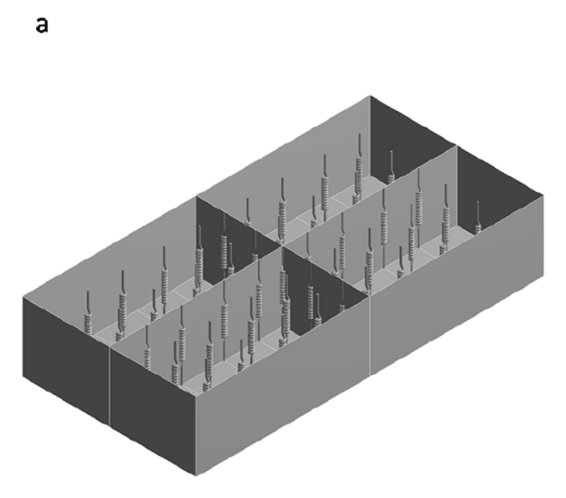

b

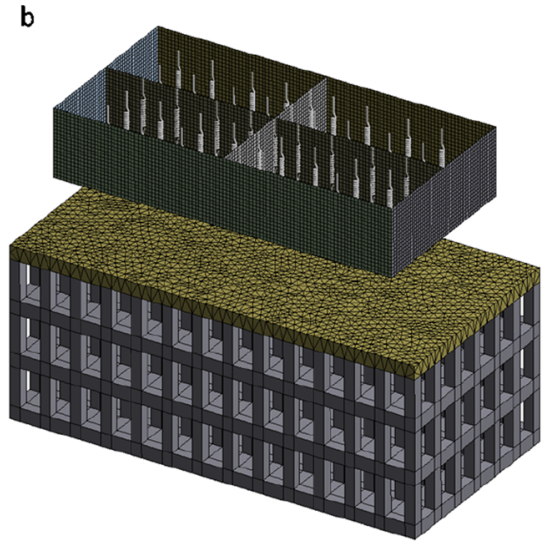


Fig. 8 Terms for deflections and inclinations

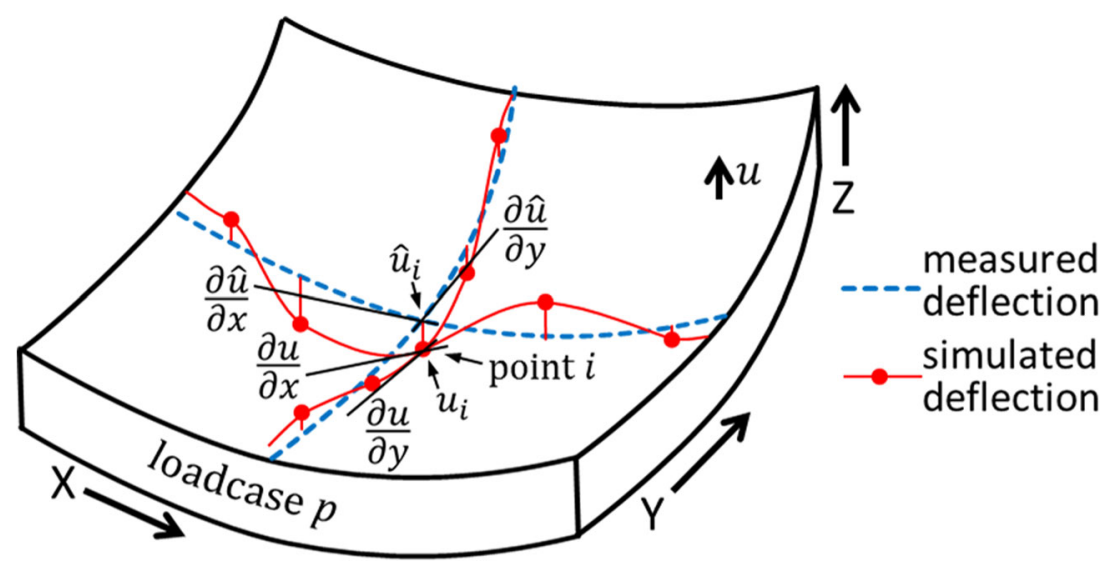

displacements will be too large since the fixed nonlinear parts are overestimated.

Another option is to leave out the nonlinear parts and to consider them separately in the simulation workflow. This leads to a reduced model always resulting in a deformation that is too small compared with the measured total displacements [13].

The most accurate option is an adaption of the basic models generated above so that nonlinear offsets are possible parallel to the linear behavior of the basic model, as presented in Fig. 9.

The non-linearities can be included in the model in two different ways. One option is the segmentation of the bolster plate so that a gap equal to the nonlinear offset opens behind the clamping surface, as shown in Fig. 9a. The contact between the two plates will close the gap under load quite realistically and the model remains easy to use due to the flat clamping surface.

The other option results from moving the nodes of the clamping surface by the amount of the nonlinear offset, and from the generation of the gap between clamping surface and forming tool, as shown in Fig. 9b. This option reduces the size of the model but increases vulnerability to misplaced boundary conditions during usage.

\section{Potential and application of the generated FE models}

\subsection{Applications and benefits}

In general, various application options result from the knowledge of the relevant elastic behavior of the forming machine described in the condensed FE models.

One basic benefit is the comparison of forming machines regarding their stiffness for an arbitrarily selected load scenario and with freely defined deflection criteria. This could also expand and qualify the current options for the formulation of target specification during an acquisition process.

An additional advantage is the possibility to generate realistic pressure distributions in a forming tool (reworked or not) on different presses and to check whether the presses are interchangeable for this specific tool in its current state. This information allows the expansion of backup strategies and increases flexibility in production planning systems.

The probably major advantage lies in the estimation of necessary crowning for the individual combination of press and forming tool in order to reduce costly rework after machining of the forming tool.

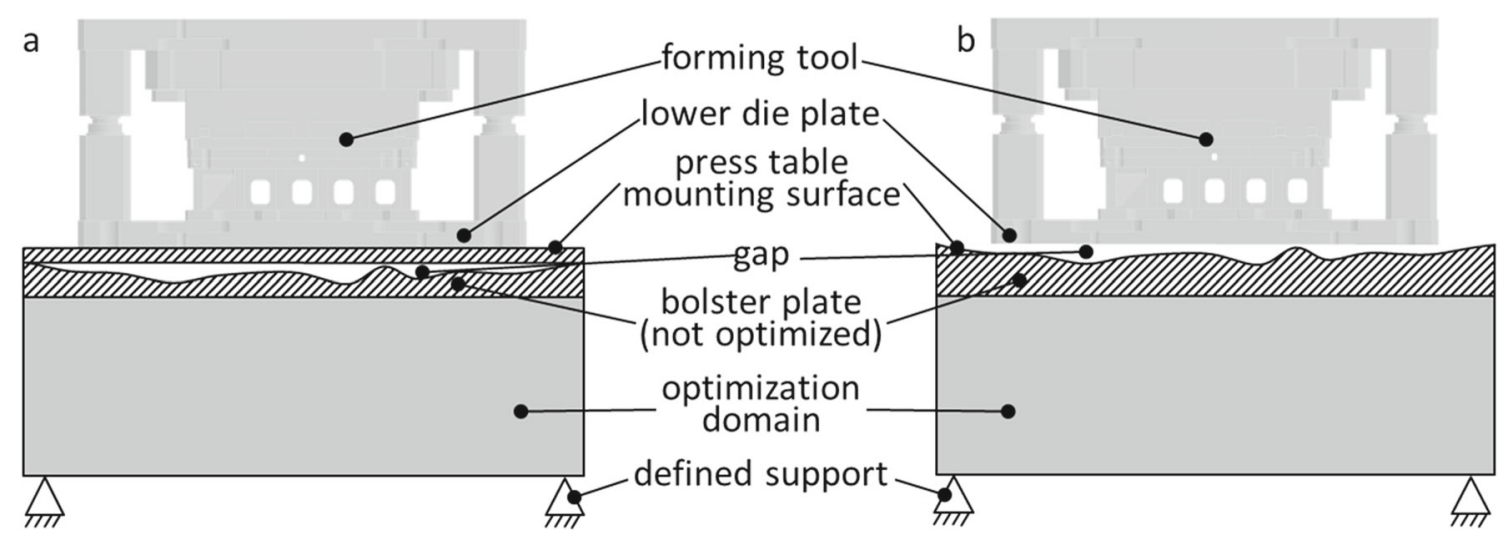

Fig. 9 Adaption of a basic model with integrated non-linearities 

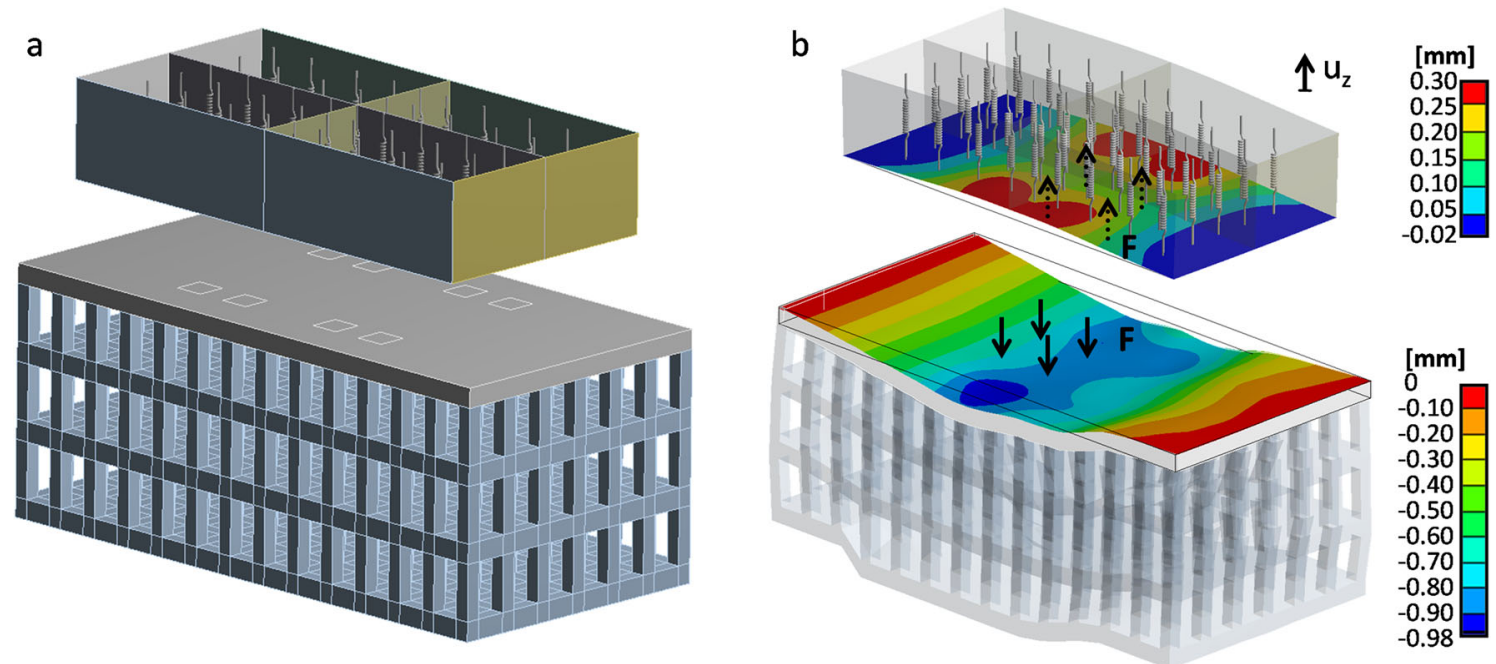

Fig. 10 Combined press model consisting of press ram and press table (a) and the elastic deflection under distributed centric load (b)

\subsection{Exemplary utilization of effective models}

In order to verify the work and its applicability, the influence of the elastic behavior of the press on the pressure distribution of a forming part is demonstrated.

Figure 10 a shows the used model consisting of press table and press ram optimized according to the measurement on the elasto-mechanical behavior of a tryout press. The calibration process of the workpiece (Fig. 11b) in the forming tool (Fig. 11a) with its load distribution leads to deflections of the press components, as shown in Fig. 10b.

For the load case presented (load of $10 \mathrm{MN}$ with 4 equal point loads in rectangle of $1400 \times 1000 \mathrm{~mm}$ ), the deflection of the clamping surfaces achieves $0.98 \mathrm{~mm}$ for the table and $0.32 \mathrm{~mm}$ for the press ram. Both values represent the resulting unevenness of the respective surface and thus are compensated with the vertical shift of the surface corners. For the press table, the mean error between FE approximation and measured displacement values is below $0.1 \mathrm{~mm}$, not only for the shown load case but for all four load cases measured including those with eccentric load. For the press ram, only centrical loads are used for fitting since the reference level of the ram will adjust to the much stiffer press table during the process. The mean error for the model of the press ram is below $0.08 \mathrm{~mm}$. Even with this remaining uncertainty, the models allow to take into account the major effect of the elastic behavior of the machine during the forming simulation.

Since the verification targets the influence of the machine's elastic behavior, no plastic deformations of the clamping surfaces are added to the used models.

\subsection{Influence of elasto-mechanical machine behavior}

Figure 12 shows the example of the resulting contact pressure between upper die and blank when pressing with a rigid ram on an elastic tool clamped to a rigid table (Fig. 12a) and when using the elastic ram and table (Fig. 12b) from the tryout press in Fig. 10.

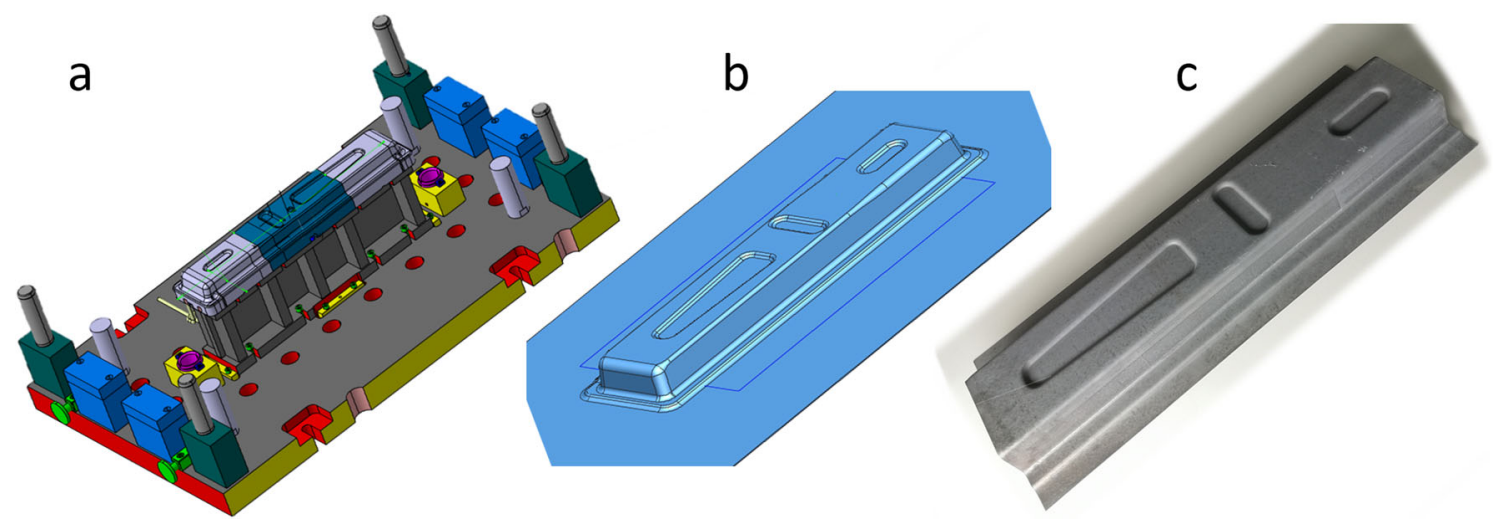

Fig. 11 Forming tool (a), simulated formed part (b), and real part formed by adjusted tool (c) for evaluation of models 
Fig. 12 Contact pressure distribution $p$ between workpiece and forming tool on rigid machine (a) and on elastic machine (b)

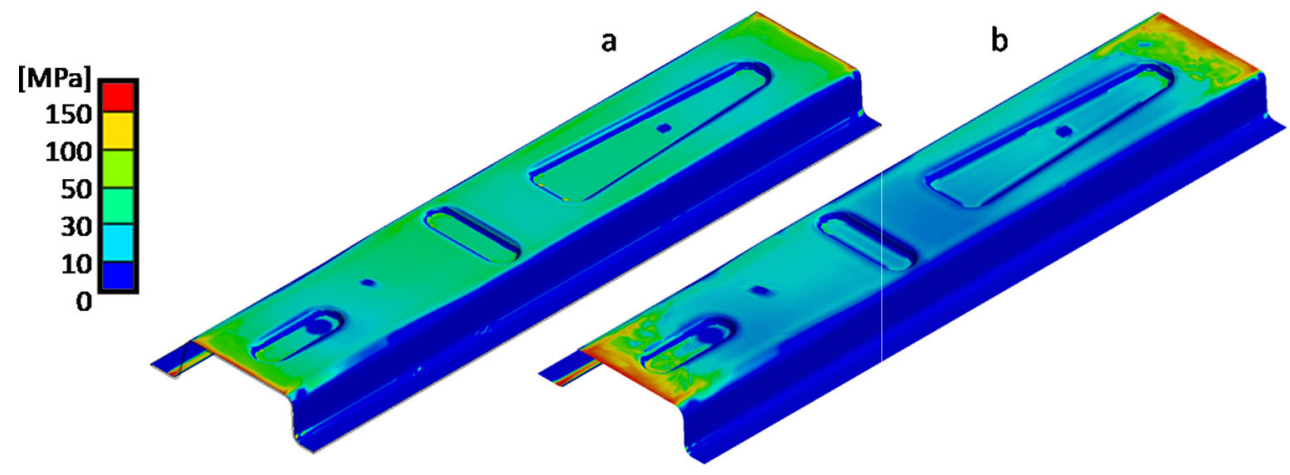

The pressure distribution from the rigid press is the desired one which the forming tool is designed for with the help of standard forming simulations. Using the forming tool on the actual elastic machine, the pressure distribution becomes uneven as expected because of the deflecting clamping surfaces of press table and press ram. This results in hotspots with overstrained, thinning and likely cracking material at the short ends of the workpiece. Sidelong, in the middle of the part, not all contours and beads are formed correctly due to lack of contact pressure.

These simulation results are intended for a qualitative comparison to show the importance of the general consideration of the machine's elasticity and the usability of the generated models presented in this paper.

Using the models of the press table and press ram, the required crowning can be estimated before the machining process of the tool. Thus, a machined tool would be already compensated for the occurring elastic deflection of the machine during the forming process.

\section{Summary and outlook}

This work addresses the importance of including the elastic behavior of the forming press into the simulation environment. For this purpose, it shows options for acquiring the elastic behavior of the relevant press components of press table and press ram via measurements. Thus, knowledge of CAD data for the individual press components is not necessary, as it typically is not available for older forming machines anyway. The measurement data is analyzed and prepared for its usage in effective FE models. Different approaches based on topological optimization as well as parametric optimization are presented for modeling. The FE models are capable of reproducing the measured elastic behavior, including nonlinearities such as flatness of clamping surfaces, within the FE simulation for inline coupling with the forming tool. The FE models optimized for an individual tryout press were used for estimating the dependence of the pressure distribution in the forming zone from the elasticity of the forming machine. Using this knowledge, saving potentials arise in the design process of forming tools, especially in the automotive industry with large car body tools.

Open Access This article is licensed under a Creative Commons Attribution 4.0 International License, which permits use, sharing, adaptation, distribution and reproduction in any medium or format, as long as you give appropriate credit to the original author(s) and the source, provide a link to the Creative Commons licence, and indicate if changes were made. The images or other third party material in this article are included in the article's Creative Commons licence, unless indicated otherwise in a credit line to the material. If material is not included in the article's Creative Commons licence and your intended use is not permitted by statutory regulation or exceeds the permitted use, you will need to obtain permission directly from the copyright holder. To view a copy of this licence, visit http://creativecommons. org/licenses/by/4.0/.

\section{References}

1. Birkert A, Haage S, Straub M (2013) Umformtechnische Herstellung komplexer Karosserieteile: Auslegung von Ziehanlagen. Springer Vieweg, Berlin

2. Brecher C, Schapp L (2007) Advances into new dimensions: coupling forming simulation with non-linear press models. In: Massivumformung, VDI-Berichte, vol 1993. VDI-Verl., Düsseldorf, pp $117-128$

3. Brecher C, Esser M, Witt S (2009) Interaction of manufacturing process and machine tool. CIRP Ann 58:588-607

4. Chen J, Xu D, Xia G, Li X, Chen J, Zhang J, Yan W, Li Y (2013) Geometric compensation for automotive stamping die design integrating structure deflection and blank thinning. Int $\mathbf{J}$ Adv Manuf Technol 66(9-12):1449-1456

5. Groche P, Hoppe F, Sinz J (2017) Stiffness of multipoint servo presses: mechanics vs. control. CIRP Ann 66(1):373-376

6. Großmann K, Luginger F, Wunderlich B, Prause M, Siegert K (2003) Kompensation der Stößelkippung mechanischer Pressen mit einem passiv-hydraulischen system. Zeitschrift für wirtschaftlichen Fabrikbetrieb 10:505-509

7. Neto DM, Coer J, Oliveira MC, Alves JL, Manach PY, Menezes LF (2016) Numerical analysis on the elastic deformation of the tools in sheet metal forming processes. Int J Solids Struct 100:270-285

8. Neugebauer R, Bouzakis KD, Denkena B, Klocke F, Sterzing A, Tekkaya AE, Wertheim R (2011) Velocity effects in metal forming and machining processes. CIRP Ann 60(2):627-650 
9. Penter L (2016) Qualifizierung von FE-Prozessmodellen zur Inbetriebnahme von Karosseriewerkzeugen Dissertation. Technische Universität Dresden, Dresden

10. Pilthammar J, Sigvant M, Hansson M, Pálsson E, Rutgersson W (2017) Characterizing the elastic behaviour of a press table through topology optimization. J Phys Conf Ser, 896

11. Pilthammar J, Sigvant M, Kao-Walter S (2018) Introduction of elastic die deformations in sheet metal forming simulations. Int $\mathbf{J}$ Solids Struct 151:76-90

12. Schongen F, Klocke F, Mattfeld P, Rjasanow S, Grzhibovskis R (2014) FEM/BEM simulation of cold forging process considering press-tool-workpiece interaction. Procedia Eng, 81
13. Tehel R, Päßler T, Mihm M (2019) Modeling elastic behavior of forming machine components to reduce tool manufacturing time. Procedia Manuf 27:177-184

14. Waltl H, Struck R (2012) Prüfwerkzeug zur Ermittlung der Eigenschaften einer Umformpresse unter Realbedingungen. Offenlegungsschrift

15. Wiebenga JH, Atzema EH, An YG, Vegter H, Van Den Boogaard AH (2014) Effect of material scatter on the plastic behavior and stretchability in sheet metal forming. J Mater Process Technol 2014(2):238-252

Publisher's note Springer Nature remains neutral with regard to jurisdictional claims in published maps and institutional affiliations. 